\title{
Synthesis of multiwalled carbon nanotubes by high-temperature vacuum annealing of amorphous carbon
}

\author{
S. Seelan, D.W. Hwang, L.-P. Hwang, A.K. Sinha* \\ Department of Chemistry, Institute of Atomic and Molecular Sciences, National Taiwan University, \\ Academia Sinica, P.O. Box 23-34, Taipei, Taiwan, Republic of China
}

Received 6 September 2003; received in revised form 14 December 2003; accepted 16 January 2004

\begin{abstract}
Vacuum annealing of a mixture of amorphous carbon and cobalt nanoparticles supported on microporous zeolite at high-temperature results in the formation of multiwalled carbon nanotubes which are essentially filled with metal nanoparticles or nanowires as observed by transmission electron microscopy. The electronic properties of nanotubes by variable temperature ESR techniques shows that $g$ values show little change with temperature from 77 to $327 \mathrm{~K}$ but the line width $\left(\Delta H_{\mathrm{pp}}\right)$ of the ESR signal for nanotubes synthesized from amorphous carbon increases from $7.9 \mathrm{G}$ at $77 \mathrm{~K}$ to $9.5 \mathrm{G}$ at $327 \mathrm{~K}$.
\end{abstract}

(C) 2004 Elsevier Ltd. All rights reserved.

Keywords: Carbon nanotube; ESR; Vacuum annealing; Zeolite

\section{Introduction}

Fascinating mechanical and electronic properties of carbon nanotubes and their high potential for application in nanoscale devices and composite materials has motivated researchers to look for better synthesis methods to improve their quality and yield. Several synthesis methods have been tried and reported such as arc discharge [1,2], laser vaporization of a graphite electrode in the presence of metal catalysts [3], pyrolysis of hydrocarbon vapor over a transition metal in-

\footnotetext{
*Corresponding author. Materials Department, Toyota Central R\&D Labs Inc., 41-1 Yokomichi, Nagakute-Cho, Aichi-Gun, Aichi 480-1192, Japan. Tel.: +81-561-63-7683; fax: +81-561-63-6137.

E-mail address: e1258@mosk.tytlabs.co.jp (A.K. Sinha).
}

corporated silica or zeolite support [4,5], pyrolysis of metallocene [6]. Intense research is also going on towards filling carbon nanotubes with metals $[5,7]$ to improve their electronic and magnetic property [7] with potential application in nanodevices and as catalysts for chemical reactions.

It has been reported earlier that catalytic carbon nanotube growth is possible using pure carbon vapor as carbon precursor rather than using a carbon-containing gas mixture under the conditions of the conventional catalytic process on a supported metal catalyst [8]. It has also been reported recently that it is possible to form gold nanowires encaged in carbon nanotubes using amorphous carbon and $\mathrm{Au}$ nanoparticles [9] by a self-assembly process. In our recent work we have reported the incorporation of cobalt nanoparticles and nanowires in multiwalled carbon nanotubes 
(MWNTs) using microporus AlPO-5 support and acetylene gas as carbon precursor [5]. Here, we report for the first time, synthesis of MWNTs with very high internal pore diameter which are filled with Co nanoparticles and nanowires, by hightemperature and low-pressure annealing of an intimate mixture of amorphous carbon and cobalt nanoparticles supported on zeolite Na-Y. Depending on the wrapping geometry, a nanotube can be either a metal or a small or large gap semiconductor $[3,7,8]$. Although such effects can be more clearly seen in case of singlewall nanotubes, nested MWNTs should also exhibit mesoscopic phenomena, since each layer is weakly coupled to its neighbors, and hence conserves its quasi-onedimensional character. So, the electronic conduction properties of these nanotubes was studied by variable temperature ESR measurements.

\section{Experimental}

The nanotube synthesis procedure was as follows: an intimate mixture of amorphous carbon and $\mathrm{Co} / \mathrm{Na}-\mathrm{Y}$ catalyst was prepared by grinding. The mixture was annealed in a sealed quartz tube at a reduced pressure $\left(0-10^{-2}\right.$ Torr) in vacuum or in hydrogen atmosphere at $723-973 \mathrm{~K}$ for specified time (30-120 min.). The supported cobalt catalyst $\mathrm{Co} / \mathrm{Na}-\mathrm{Y}$, was prepared by porous impregnation method by adding zeolite $\mathrm{Na}-\mathrm{Y}$ to a stirring solution of cobalt nitrate $(5 \mathrm{wt} \%)$ in water at $335 \mathrm{~K}$. The resulting suspension was stirred to dryness, then dried overnight in oven at $413 \mathrm{~K}$ and finally ground to obtain fine supported catalyst powder (100 mesh). Conventional synthesis of MWNTs was done using the earlier reported procedure [5]. A typical synthesis involved decomposition of acetylene $(10 \mathrm{ml} / \mathrm{min})$ diluted with nitrogen $(100 \mathrm{ml} / \mathrm{min})$ for $90 \mathrm{~min}$ over cobalt catalyst supported on zeolite $\mathrm{Na}-\mathrm{Y}(\mathrm{Si} / \mathrm{Al}=49.5$, P.Q. Zeolites, Holland) placed in a ceramic boat in a quartz tubular reactor at $973 \mathrm{~K}$.

The formation of carbon nanotubes was studied by transmission electron microscopy (TEM) (JEOL 2000FX, $120 \mathrm{kV}$ ) after sonicating the samples in ethanol and dispersing a drop of the solution over holey carbon grids. The carbon nanotubes were purified before ESR measurements according to a literature procedure [10]. The raw sample was first ultra-sonicated to disperse the MWNTs, followed by immersion in bromine water at $90^{\circ} \mathrm{C}$ for $3 \mathrm{~h}$. The residue obtained was heated in air at $520^{\circ} \mathrm{C}$ for $45 \mathrm{~min}$. The black product was first treated with $20 \% \mathrm{HF}$ solution to dissolve the $\mathrm{Na}-\mathrm{Y}$ support followed by treatment with $6 \mathrm{~N}$ $\mathrm{HNO}_{3}$ solution to remove cobalt metal nanoparticles from the samples.

\section{Results and discussions}

Carbon nanotube formation could be observed by annealing under vaccum $\left(10^{-2}\right.$ Torr $)$ the mixture of amorphous carbon and $\mathrm{Co} / \mathrm{Na}-\mathrm{Y}$ catalyst. At $723 \mathrm{~K}$ and $10^{-2}$ Torr we observe the formation of few short and thick walled tubes with very narrow pores. When annealing temperature was increased to $973 \mathrm{~K}$ we could still observe only a few ill-formed nanotubes and nanofibres attached to the support surface. But when the annealing was carried out at $973 \mathrm{~K}$ in $\mathrm{H}_{2}$ atmosphere and reduced pressure $(\sim 0$ Torr $)$ for $90 \mathrm{~min}$, we observed the formation of network of long carbon nanotubes with very thin walls, having an average outer diameter of $20 \mathrm{~nm}$ and inner pore diameter was $10-15 \mathrm{~nm}$ (Fig. 1a). Formation of few single wall carbon nanotubes could also be observed. High magnification TEM invariably showed the presence of metal particles (Fig. 2a) or metal nanowires (Fig. 2b) inside the tubes. In addition, some metal nanoparticles encapsulated in carbon microspheres were also observed. Presence of metal particles of different shapes and sizes suggests that the nanotube growth is catalyzed by metal particles and as the tubes grow the metal particles get trapped inside the growing nanotubes.

Carbon nanotubes grown conventionally by pyrolysis of acetylene did not show presence of metal particles either at the base, tip or in the body of the tubes (Fig. 1b) which is contrary to the results for the MWNTs grown from amorphous carbon (Fig. 1a). Metal filling of carbon filaments produced by pyrolysis of various hydrocarbons has only sometimes been observed [10]. But in the case of nanotubes produced from amorphous 

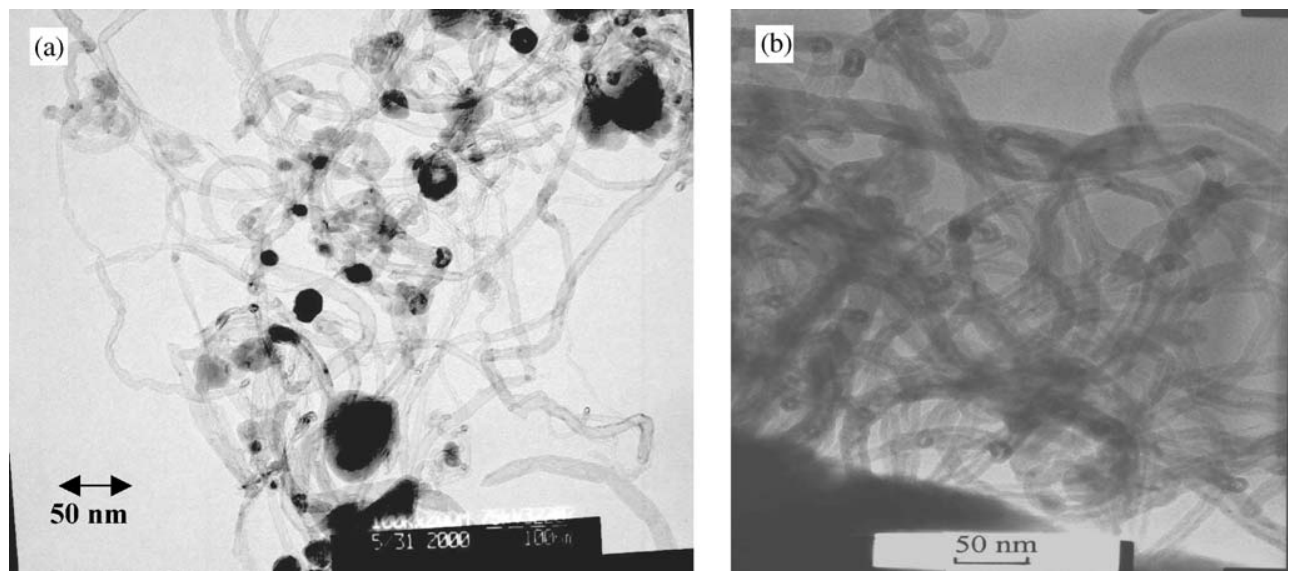

Fig. 1. TEM image of carbon nanotubes synthesized from: (a) amorphous carbon and Co nanoparticles supported on zeolite Na-Y at $973 \mathrm{~K}, \sim 0$ Torr $\mathrm{H}_{2}$ atmosphere and (b) acetylene and Co nanoparticles supported on zeolite Na-Y at $973 \mathrm{~K}$.
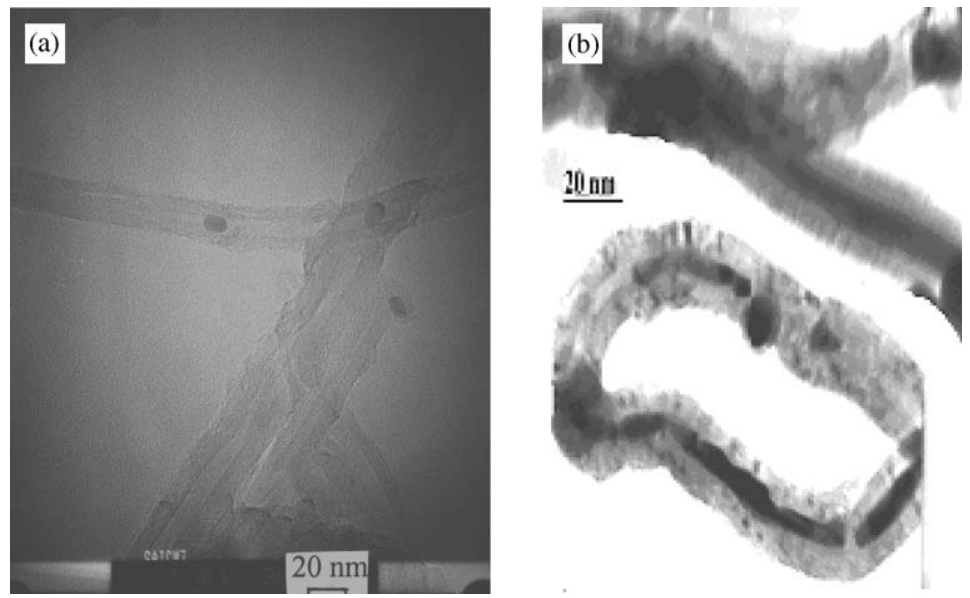

Fig. 2. High magnification TEM image of carbon nanotube synthesized from amorphous carbon shows clearly the presence of cobalt nanoparticles (a) and nanowires (b) inside the tubes.

carbon we invariably see the presence of metal particles inside the nanotubes, which implies that unlike the case of the conventional synthesis the presence of metal is essential for the nanotube growth when using amorphous carbon as carbon source. The inner diameter of the nanotubes from amorphous carbon is higher $(10-15 \mathrm{~nm})$ that for the conventional nanotubes $(5-9 \mathrm{~nm})$. The differences in the synthesis condition as well as the difference in the nature of carbon source is probably responsible for the differences observed. The growth of nanotubes using two different synthesis methods would probably follow different growth mechanisms. High temperature and low pressure for nanotube synthesis using amorphous carbon could favor the formation of highly mobile metal-carbon solid solution resulting in the encapsulation of metal particles inside the nanotubes as well as the formation of encaged metal nanowires (Fig. 3). Liquid like nature of $\mathrm{Co}$ 
(a)

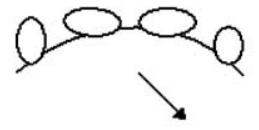

(b)

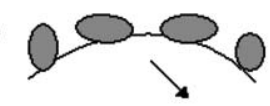

(c)

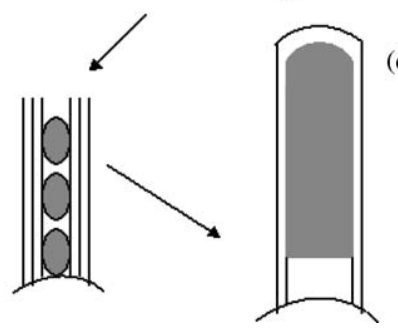

Fig. 3. Probable growth stages of carbon nanotubes from amorphous carbon and $\mathrm{Co} / \mathrm{Na}-\mathrm{Y}$ : Cobalt nanoparticles on the $\mathrm{Na}-\mathrm{Y}$ support surface (a), form super-saturated metal-carbon solid solution at high-temperature and low-pressure conditions (b), leading to the precipitation of carbon nanotubes around cobalt nanoparticles (c) and nanowires (d).

nanoparticles during nanotube growth could explain the presence of Co metal inside the carbon nanotubes synthesized from amorphous carbon. While in the case of conventionally growing nanotubes (Fig. 1b) the metal particles remain embedded in the support during the nanotube growth process making the tubes metal free [5]. It has been speculated earlier that the metal particles must have the properties of a liquid during the initial stage of growth [11] and liquid nanoparticles of catalyst initiate the growth of carbon nanotubes $[12,13]$.

The study of carbon nanotubes by ESR can give useful indications of the behavior of conduction electrons in order to predict whether metallic or narrow gap semiconducting tubes are present. The MWNTs synthesized using amorphous carbon were purified to remove Co nanoparticles to compare their electronic conduction properties at different temperatures using variable temperature ESR studies. The as-synthesized MWNT samples show a very broad and intense ESR signal $\left(\Delta H_{\mathrm{pp}}\right.$ $\sim 800 \mathrm{G})$ due to the Co catalyst particles and it masks any signal due to MWNTs. The samples after purification show free electron $g$ value of 2.004 at room temperature and the $g$ values show little change with temperature from 77 to $327 \mathrm{~K}$ (Fig. 4). Variable temperature ESR studies also

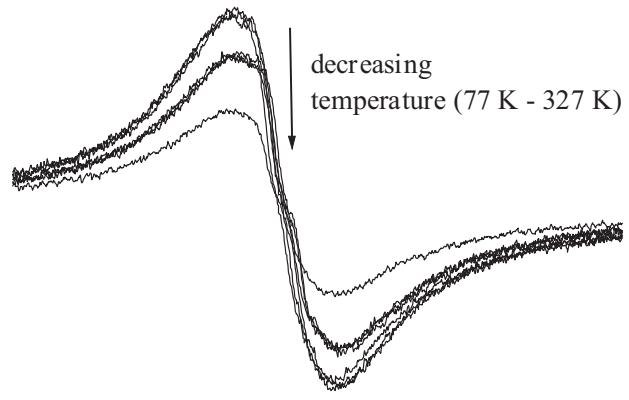

Fig. 4. Variable temperature (77-325 K) ESR spectra of carbon nanotubes synthesized from amorphous carbon and Co nanoparticles supported on zeolite Na-Y at $973 \mathrm{~K}, \sim 0$ Torr $\mathrm{H}_{2}$ atmosphere.

show that the line width $\left(\Delta H_{\mathrm{pp}}\right)$ of the ESR signal for nanotubes synthesized from amorphous carbon increases from $7.9 \mathrm{G}$ at $77 \mathrm{~K}$ to $9.5 \mathrm{G}$ at $327 \mathrm{~K}$. In case of carbon nanotubes prepared using amorphous carbon, cobalt nanoparticles which are invariably present inside the tubes may contribute to spin relaxation and hence variation in line width $\left(\Delta H_{\mathrm{pp}}\right)$ with temperature is observed. The ESR spectra are symmetric in nature which could be due to the size of conducting particles in the purified samples being comparable to the skin depth (at $10 \mathrm{GHz}$ ) of the MWNTs [14]. Previous ESR measurement on oriented MWNT [15] has also yielded a symmetric line whose magnitude and anisotropy of $g$, and whose temperature dependence were consistent with small graphite-like semimetallic particles.

\section{Conclusions}

In conclusion, we have been able to develop a new and potentially useful method for growing carbon nanotubes with high inner diameter and which are filled with metal nanoparticles and nanowires, by annealing amorphous carbon and supported cobalt nanoparticles at high temperature and low pressure in hydrogen atmosphere. These nanotubes could be expected to have interesting electronic and magnetic properties. The growth of these nanotubes may follow a mechanism different from that for the nanotubes grown by pyrolysis of acetylene over supported metal 
catalysts, which produces nanotubes free of metal particles and with lower inner diameters. Even though both the samples show almost similar $g$ values, the variation in line width $\left(\Delta H_{\mathrm{pp}}\right)$ with temperature for the MWNTs synthesized from amorphous carbon is more than that for nanotubes synthesized using acetylene which can be attributed to difference in morphology for the two MWNT samples as well as to the presence of Co nanoparticles in the former sample.

\section{References}

[1] Iijima S. Nature (London) 1991;354:56-8.

[2] Journet C, Maser WK, Bernier P, Loiseau A, Lamy de la Chappelle M, Lefrant S, Deniard P, Lee R, Fischer JE. Nature (London) 1997;388:756-8.

[3] Thess A, Lee R, Nikolaev P, Dai P, Petit P, Robert J, Xu C, Lee YH, Kim SG, Rinzler AG, Colbert DT, Scuseria GE, Tomanek D, Fisher JE, Smalley RE. Science 1996;273:483-7.
[4] Piedigrosso P, Konya Z, Colomer J-F, Fonseca A, Tendeloo GV, Nagy JB. Phys Chem Chem Phys 2000; 2:163-70.

[5] Sinha AK, Hwang DW, Hwang LP. Chem Phys Lett 2000;332:455.

[6] Sen R, Govindraj A, Rao CNR. Chem Phys Lett 1997;267:276-80.

[7] Nolan PE, Cutler AH, Lynch DG. US Patent 1998;US 5780101.

[8] Kukovitsky EF, L’vov SG, Sainovn NA. Chem Phys Lett 2000;317:65-70.

[9] Oku T, Suganuma K. Chem Comm 1999; 2355-66.

[10] Hou PX, Bai S, Yang QH, Liu C, Cheng HM. Carbon 2002;40:81.

[11] Bernardo CA, Alstrup I, Rostrup-Nielsen R. J Catal 1985;96:517.

[12] Oberlin A, Endo M, Koyama T. J Cryst Growth 1976; 32:335.

[13] Kukovitsky EF, L'vov SG, Sainov NA. Mol Mater 1998; 10:165.

[14] Petit P, Jouguelet E, Fischer JE, Rinzler AG, Smalley RE. Phys Rev B 1997;56:9275.

[15] Chauvet O, Forro L, Bacsa W, Ugarte D, Doudin B, de Heer WA. Phys Rev B 1995;52:R6963. 